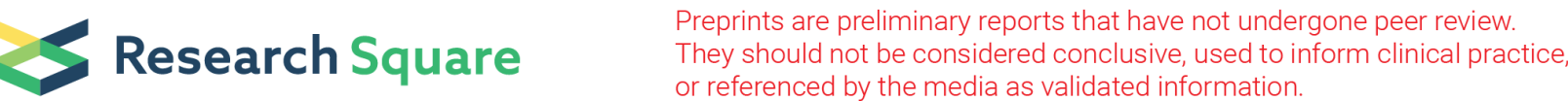

\section{Folic Acid Can Reduce Hyperhomocysteinemia- induced Vascular Damage by Immune/Inflammatory Response in Spontaneously Hypertensive Rats}

\section{Lihua Zhang}

Department of Medicine

\section{Zhongliang li}

Jinan maternity and child care hospital affiliated to shandong first medical university https://orcid.org/0000-0002-7599-9328

\section{Changcheng Xing}

Department of Cardiology

\section{Ning Gao}

Cheeloo College of Medicine

\section{Ping Cao}

Cheeloo College of Medicine

Qingcheng Hui

Department of Cardiology

\section{Lin Yun}

Department of Medicine

Rui Xu ( $\nabla$ xuruicn@hotmail.com )

Cheeloo College of Medicine https://orcid.org/0000-0001-5030-2451

\section{Research}

Keywords: Hypertension, hyperhomocysteinemia, folic acid, NADPH oxidase, NF-kB, arterial injury

Posted Date: May 15th, 2020

DOl: https://doi.org/10.21203/rs.3.rs-25948/v1

License: (c) (1) This work is licensed under a Creative Commons Attribution 4.0 International License. Read Full License 


\section{Abstract \\ Background}

Hypertension associated with hyperhomocysteinemia (HHcy) is correlated with a high risk of vascular diseases. However, the mechanisms of HHcy-associated hypertensive vascular damage and the efficacy of folic acid (FA) as a treatment have not been fully elucidated. The aim of the present study was to evaluate the role of immune/inflammatory molecules and oxidizing factors in HHcy-associated hypertensive vascular damage, and to observe the intervention effect of FA on the two vascular injury factors.

\section{Methods}

Wistar-Kyoto rats (WKYs) and spontaneously hypertensive rats (SHRs) were administered DL-Hcy intraperitoneally to mimic HHcy and hypertension associated with HHcy for 12 weeks. WKYs and SHRs were randomized into WKY group, HHcy group, SHR group, SHR + HHcy group and SHR + HHcy + FA group. Mean tail artery blood pressure, plasma Hcy, serum SOD and MDA of rats in each group were compared. The thoracic aorta and bilateral carotid artery of rats were harvested for morphometric and immunostaining analyses. Quantitative real-time polymerase chain reaction (qRT-PCR) and Western blot were used to detect the expression of immune/inflammatory molecules such as TNF-a, IL-6, NF-KB p65/Rela and NF-KB2 and oxidative factors such as Nox2 and Nox4.

\section{Results}

We found that vascular inflammatory factors of vascular adhesion protein-1 (VAP-1), interleukin-6 (IL-6), and nuclear factor-k-gene binding (NF-kB) p65/Rela in HHcy-associated hypertensive rats were significantly higher than those in SHRs (Pख0.05). While the oxidative stress indicators of Nox2 and Nox4 in HHcy-associated hypertensive rats were not significantly higher than those in SHRs (Pख0.05). Compared with SHRs, FA intervention in $\mathrm{HH} c y$-associated hypertensive rats significantly increased serum superoxide dismutase (SOD) levels $(P=0.000)$ and significantly reduced vascular inflammatory factors of IL-6 and NF-KB p65/Rela (Pख0.05), but did not significantly change the oxidative stress indicators of Nox2 and Nox4 (Pख0.05).

\section{Conclusions}

$\mathrm{HHcy}$-induced immune/inflammatory response plays a dominant role in vascular damage of HHcyassociated hypertensive rats. In addition to reducing the negative effects of HHcy, FA might involve unique antioxidant effects and inhibition of immune/inflammatory overreaction for HHcy-associated hypertensive rats. 


\section{Background}

Hyperhomocysteinemia (HHcy) is defined as a homocysteine (Hcy) level above $10 \mu \mathrm{mol} / \mathrm{L}$ in human blood $^{[1]}$. And hypertension associated with HHcy accounts for $75 \%$ of hypertension cases in China ${ }^{[2]}$. With the ageing of society and the decrease in the dietary intake of fresh vegetables, the incidence of hypertension associated with HHcy remains high. HHcy, known as the "new cholesterol of the 21st century", is an independent risk factor for cardiovascular and cerebrovascular diseases ${ }^{[3]}$.

Hypertension is known to be associated with increased vascular inflammation ${ }^{[4]}$. Patients of hypertension associated with $\mathrm{HHcy}$ have a higher incidence of vascular events and disability ${ }^{[5]}$ than those of pure hypertension, suggesting that HHcy can aggravate vascular inflammation in hypertension. Previous studies indicate that HHcy can decrease endothelium-dependent and blood flow-mediated vasodilation ${ }^{[6]}$, increase oxidative stress ${ }^{[7-9]}$ and inflammatory reactions ${ }^{[10,11]}$, and is closely related to the thickening of the arterial intima-media ${ }^{[12]}$ and intravascular thrombosis ${ }^{[13]}$. HHcy is the abnormal aggregation of Hcy that is an intermediate product linking methionine metabolism and transsulfuration ${ }^{[14]}$ in the body. Therefore, HHcy can be regarded as the marker of abnormal methionine and / or abnormal trans-sulfur metabolism in the body. Additionally, Hcy unique structure can interfere with amino acid metabolism and form Hcy-proteins affecting protein structure and function ${ }^{[15-17]}$. Hcy is also involved in FA metabolism and affects methylation ${ }^{[18]}$. Therefore, we hypothesize that the pathological mechanism of HHcy is manifested by more Hcy-proteins triggering an overreaction of the immune system, which leads to acute arterial dysfunction and chronic structural change in hypertension associated with HHcy.

FA is generally used to lower HHcy and is a component of anti-hypertensive drug combinations for hypertension associated with $\mathrm{HHcy}^{[2]}$. FA plays a key role as a donor of single carbons in DNA synthesis and repair ${ }^{[19]}$. As an important methyl donor, FA also participates in the processes of Hcy remethylation to form methionine ${ }^{[14]}$, indirectly affecting gene expression, protease function, and disease status through methylation. FA is also a powerful antioxidant ${ }^{[20]}$ and closely associated with immune cells that highly express FA receptors ${ }^{[21,22]}$. Studies have found that FA supplementation can reduce the risk of cardiovascular and cerebrovascular events ${ }^{[23]}$. Therefore, we hypothesize that the vasoprotective mechanism of FA, in addition to reducing the negative effects of HHcy, also involves antioxidant effects and inhibition of immune/inflammatory overreaction.

In this study, normal blood pressure Wistar-Kyoto rats (WKYs) and spontaneously hypertensive rats (SHRs) were administered DL-Hcy intraperitoneally to mimic human HHcy and hypertension associated with $\mathrm{HHcy}{ }^{[24]}$. Vascular expression of immune/inflammatory molecules such as nuclear factor-k-gene binding (NF-KB), tumour necrosis factor-alpha (TNF-a), interleukin-6 (IL-6), vascular adhesion protein-1 (VAP-1), monocyte chemotactic protein 1 (MCP-1), and CD68 and oxidative factors nicotinamide adenine dinucleotide phosphate (NADPH) oxidase (Nox) such as Nox2 and Nox4 were measured and compared, in order to gain insights on the immune/inflammatory mechanism of severe vascular damage in 
hypertension associated with HHcy and the protective effects of FA on hypertension associated with HHcy through inhibiting oxidative stress and excessive immune responses.

\section{Materials And Methods}

\subsection{Animals}

Animal care and experimental protocol for this study were approved by the Committee on the Use of Live Animals in Teaching and Research of Qianfoshan Hospital. The Laboratory Animal Unit of Qianfoshan Hospital is fully accredited by the Association for Assessment and Accreditation for Laboratory Animal Care (AAALAC International). Sixteen healthy male SPF WKYs and 24 male SHRs, (250-270 g, 12 weeks) were provided from the Beijing Vital River Laboratory Animal Center (Beijing, China) and had free access to chow and water. The animal room was controlled at a constant temperature $\left(22 \pm 2{ }^{\circ} \mathrm{C}\right)$, humidity, and $12 \mathrm{~h}$ light/dark cycle.

\subsection{Experimental grouping}

WKYs were randomly distributed into two experimental groups: WKY group and HHcy group, 8 rats in each group; SHRs were randomly distributed into three experimental groups: SHR group, HHcy + SHR group and HHcy + SHR + FA group, 8 rats in each group. WKY group and SHR group were administered physiological saline (PS, $5 \mathrm{ml} / \mathrm{kg}$, twice a day) intraperitoneally for 12 weeks; HHcy group, HHcy + SHR group and HHcy + SHR + FA group were injected intraperitoneally with $2 \%$ DL-Hcy (5 ml/kg, twice a day, H4628, Sigma-Aldrich, St. Louis, USA) for 12 weeks. At the same time during the last 8 weeks of the experiment, HHcy + SHR + FA group was given FA $(0.4 \mathrm{mg} / \mathrm{kg} / \mathrm{d}$, F7876, Sigma) by gavage; and the other four groups were given gavage of the same amount of PS. FA was freshly dissolved in $0.5 \mathrm{ml}$ PS immediately before the experiment.

\subsection{Blood pressure measurement}

Blood pressure was measured by a tail-cuff method using non-invasive rat tail artery manometer (Beijing Ruolong Biotechnology Company, BP-2010A). At least three measurements were taken for each animal, each measurement was more than 5 minutes, and the mean of three measurements was calculated as the ultimate blood pressure.

\subsection{Specimen collection}

The rats were anesthetized by intraperitoneal injection of $10 \%$ chloral hydrate $(0.3 \mathrm{ml} / 100 \mathrm{~g})$ to collect blood from vena cava for measuring Hcy, SOD and MDA, then to harvest the thoracic aorta and bilateral carotid artery. Part of the arterial tissue was prepared by standard methods for morphometric and immunostaining analyses. And the remaining arterial tissue was immediately frozen in liquid nitrogen and stored at $-80^{\circ} \mathrm{C}$ for measuring protein level by Western blotting and semiquantification of mRNA expression by quantitative real-time polymerase chain reaction (qRT-PCR). 


\subsection{Measurement of Hcy, MDA and SOD}

The blood was drawn from vena cava of rats and sent to the laboratory of Qianfoshan Hospital. The concentration of plasma Hcy was measured by using a Cobas 8000 automatic biochemistry analyzer (Roche, Switzerland). The activity of serum SOD and the level of serum MDA were determined using commercial kits (Jiancheng Institute of Biological Technology, Nanjing, Jiangsu, China) according to the manufacturer's instruction.

\subsection{Histopathology and immunohistochemistry}

Five-micron sections of formalin fixed, paraffin-embedded aorta and carotid were stained with hematoxylin and masson to assess vascular pathology, vessel wall thickness and collagen deposition. Each sample slice was observed under the microscope (Olympus, Tokyo, Japan) at a magnification of $200 \times$.

Immunohistochemistry analysis for CD68 and VAP-1 was performed according to the manufacturer's instructions. Antigen retrieval was performed on serial artery sections and incubated with antibodies against CD68 (1:100, Wuhan Three Eagles, GB11067) and VAP-1 (1:100, Proteintech, ab181168), then the artery sections were incubated with a fluorescent secondary antibody. The protein expression of CD68 and VAP-1 were assessed on a Nikon Eclipse Ti-E inverted epi-fluorescent microscope (Nikon Instruments, Tokyo Japan). Brown areas were considered positive. And immunohistochemistry analysis for CD68 and VAP-1 was performed by Image-pro plus 6.0 (Media Cybernetics, Inc, Rockville, MD, USA).

\section{7 qRT-PCR}

Total RNA was extracted from the homogenate of fresh-frozen thoracic aorta without adipose tissue using TRIzol reagents (Invitrogen, 15596026). The RNA was quantified spectrophotometrically (Spectrophotometer, Merinton, SMA4000), and two micrograms of RNA was reverse transcribed into cDNA with RT reagent kit with gDNA Eraser (Takara, RR047A) for qRT-PCR. The CDNAs of MCP-1, VAP-1, TNF-a, IL-6, NF-kB p65/Rela, NF-kB2, Nox2, Nox4, transforming growth factor (TGF)- $\beta 1$ and TGF- $\beta 3$ were used to determine gene expression using TB Green Premix Ex Taq (Takara, RR820A) in a real-time PCR machine (ABI ViiA 7, Applied Biosystems, Foster City, CA). And the program was run with reaction cycling of initial denaturing $\left(95^{\circ} \mathrm{C}, 30 \mathrm{~s}\right)$, followed by 40 cycles of denaturing $\left(95^{\circ} \mathrm{C}, 5 \mathrm{~s}\right)$ and extension $\left(60^{\circ} \mathrm{C}\right.$, $30 \mathrm{~s}$ ). A melting curve was run to confirm specificity of PCR products. Gene expression levels were normalized to GAPDH expression levels. The relative quantity of mRNA expression was calculated according to the cycle threshold $\left(2^{-\Delta \Delta C t}\right)$ method. Target genes for amplification are listed in Table 1 .

\subsection{Western blotting}

Total proteins were extracted from the homogenate of fresh-frozen thoracic aorta without adipose tissue using Protein Extraction Kit (invent, SA-03-BV). Protein samples (30 mg per lane) were separated by SDSPAGE and transferred to PVDF membrane. The PVDF membrane was blocked with 5\% milk in Tris- 
buffered saline Tween and incubated with primary antibodies (Anti-TNF-a, PTG, 17590-1-AP; Anti-IL-6, PTG, 21865-1-AP; Anti-NOX2, abcam, ab129068) overnight at $4^{\circ} \mathrm{C}$. Then the PVDF membranes were washed with Tris-buffered saline Tween solution and incubated with horseradish peroxidase conjugated second antibody for $1 \mathrm{hr}$. ChemiDoc ${ }^{\mathrm{TM}}$ Touch Gel imaging system (Bio-Rad, Hercules, CA, USA) was used to visualize immunoreactivity with a chemiluminescent HRP substrate (Vazyme, Nanjing, Jiangsu, China). The band intensities were determined using Image Lab software and expressed relative to GAPDH.

\subsection{Statistical analysis}

Statistical analysis was conducted using one-way ANOVA followed by LSD test. Results were expressed as means \pm SE. All statistical analyses were performed using the SPSS software version 13.0. $P<0.05$ was considered statistically significant.

\section{Results}

\subsection{Comparison of Hcy between groups}

After intraperitoneal injection of DL-Hcy, levels of plasma Hcy were significantly increased in the HHcy group rats when compared with WKYs (HHcy, $25.48 \pm 2.01 \mu \mathrm{mol} / \mathrm{L} ; \mathrm{WKY}, 6.30 \pm 1.47 \mu \mathrm{mol} / \mathrm{L} ; \mathrm{P}=0.000, \mathrm{n}$ = 8); Levels of serum Hcy were also significantly increased in HHcy + SHR group when compared with SHR group (HHcy +SHR, $27.61 \pm 1.53 \mu \mathrm{mol} / \mathrm{L} ; \mathrm{SHR}, 8.26 \pm 1.77 \mu \mathrm{mol} / \mathrm{L} ; \mathrm{P}=0.000, \mathrm{n}=8)$. The Hcy levels in both HHcy group and HHcy + SHR group were more than $25 \mu \mathrm{mol} / \mathrm{L}$, which fully complied with the HHcy standard ${ }^{[1]}$ and could be used to mimic hypertension associated with HHcy for experimental research. After FA treatment, there was a marked reduction in the Hcy levels in HHcy + SHR + FA group when compared with the HHcy + SHR group rats (HHcy + SHR + FA group, $10.93 \pm 2.34 \mu \mathrm{mol} / \mathrm{L}$ vs HHcy $+\mathrm{SHR}$ group, $27.61 \pm 1.53 \mu \mathrm{mol} / \mathrm{L} ; \mathrm{P}=0.000, \mathrm{n}=8$ ); But there was still a significant increase in the Hcy levels in $\mathrm{HHcy}+\mathrm{SHR}+\mathrm{FA}$ group when compared with SHR group $(\mathrm{HHcy}+\mathrm{SHR}+\mathrm{FA}$ group, $10.93 \pm 2.34 \mu \mathrm{mol} / \mathrm{L} \mathrm{vs}$ SHR group, $8.26 \pm 1.77 \mu \mathrm{mol} / \mathrm{L} ; \mathrm{P}=0.007, \mathrm{n}=8$ ). (Fig. 1-A, Table 2)

\subsection{Comparison of blood pressure between groups}

After intraperitoneal injection of DL-Hcy, the systolic and diastolic pressures of HHcy rats were slightly higher than those of WKYs, but the differences between the two groups were not statistically significant (SBP, $121.88 \pm 9.89 \mathrm{mmHg}$ vs $119.25 \pm 11.85 \mathrm{mmHg}$; DBP, $86.63 \pm 6.80 \mathrm{mmHg}$ vs $81.13 \pm 4.32 \mathrm{mmHg}$; > $0.05, n=8)$; The systolic and diastolic pressures of HHcy-associated hypertensive rats were also slightly higher than those of SHRs, but the differences between the two groups were also not statistically significant (SBP, $209.75 \pm 9.81 \mathrm{mmHg}$ vs $205.13 \pm 9.54 \mathrm{mmHg}$; DBP, $140.63 \pm 7.39 \mathrm{mmHg}$ vs $134.88 \pm$ $6.73 \mathrm{mmHg} P>0.05, n=8)$. After $F A$ treatment, there was no significant change in blood pressure between HHcy + SHR + FA group and HHcy + SHR group. (Fig. 1-B, Table 2)

\subsection{Comparison of serum MDA and SOD between groups}


After intraperitoneal injection of DL-Hcy, levels of serum MDA were significantly increased in HHcy group rats when compared with those in WKYs (HHcy, $6.50 \pm 1.07 \mathrm{nmol} / \mathrm{mL}$; WKY, $5.37 \pm 0.85 \mathrm{nmol} / \mathrm{mL} ; \mathrm{P}=$ $0.026, n=8$ ); Levels of serum MDA were also significantly increased in HHcy-associated hypertensive rats when compared with those in SHRs (HHcy + SHR, $9.11 \pm 1.12 \mathrm{nmol} / \mathrm{mL}$; SHR, $7.63 \pm 0.70 \mathrm{nmol} / \mathrm{mL} ; \mathrm{P}=$ $0.004, \mathrm{n}=8)$. But there was a marked reduction in the MDA levels in HHcy + SHR + FA group when compared with HHcy + SHR group (HHcy + SHR + FA group, $6.72 \pm 1.05 \mathrm{nmol} / \mathrm{mL}$ vs HHcy + SHR group, $9.11 \pm 1.12 \mathrm{nmol} / \mathrm{mL} ; \mathrm{P}=0.000, \mathrm{n}=8$ ). (Fig. 1-C, Table 2)

After intraperitoneal injection of DL-Hcy, levels of serum SOD were significantly lower in HHcy group rats when compared with those in WKYs (HHcy, $479.64 \pm 18.38 \mathrm{U} / \mathrm{mL}$; WKY, $516.85 \pm 28.69 \mathrm{U} / \mathrm{mL} ; \mathrm{P}=0.004, \mathrm{n}$ = 8); Levels of serum SOD were also significantly lower in HHcy-associated hypertensive rats when compared with those in SHRs (HHcy-associated hypertensive rats, $362.73 \pm 30.23 \mathrm{U} / \mathrm{mL}$; SHRs, $432.50 \pm$ $19.73 \mathrm{U} / \mathrm{mL} ; \mathrm{P}=0.000, \mathrm{n}=8$ ). But there was a marked improvement in the SOD levels in HHcy $+\mathrm{SHR}+\mathrm{FA}$ group when compared with HHcy + SHR group (HHcy + SHR + FA group, $479.04 \pm 20.62 \mathrm{U} / \mathrm{mL}$ vs. HHcy + SHR group, $362.73 \pm 30.23 \mathrm{U} / \mathrm{mL} ; \mathrm{P}=0.000, n=8)$. Even the SOD levels of HHcy $+\mathrm{SHR}+F A$ group were higher than those of SHR group (HHcy + SHR + FA group, $479.04 \pm 20.62 \mathrm{U} / \mathrm{mL}$ vs SHR group, $432.50 \pm$ $19.73 \mathrm{U} / \mathrm{mL} ; \mathrm{P}=0.000, \mathrm{n}=8$ ). (Fig. 1-D, Table 2)

\subsection{Comparison of vascular pathological changes between groups}

$\mathrm{HE}$ and masson staining of arterial vascular pathology in HHcy-associated hypertensive rats showed changes, such as the thickening of the vascular smooth muscle layer, the deposition of collagen fibers, matte of vascular intima and shedding of endothelial cells; The above pathological damage was slightly mitigated by the FA treatment $(P>0.05)$ (Fig. 2-A). Immunohistochemistry of arterial vessels in HHcyassociated hypertensive rats revealed that $\mathrm{CD} 68$ cell counts were slightly more than those in other groups $(P>0.05)$ (Fig. 2-B); And the average optical $(A O)$ value of VAP-1 was significantly increased in HHcyassociated hypertensive rats compared with that in other groups $(P<0.05)$. But the AO value of VAP-1 was significantly reduced in HHcy + SHR + FA group $(P=0.001)$. (Fig. 2-C)

\subsection{Comparison of immune inflammation and oxidative stress indicators between groups}

Levels of the mRNA relative expression of immune inflammatory molecules such as TNF-a, IL-6, NF-KB p65 / Rela, and NF-KB 2 , especially IL-6 and NF-KB p65 / Rela were significantly increased in arterial tissue homogenate of HHcy-associated hypertensive rats $(P<0.05)$. But FA treatment group showed significant reductions in the mRNA levels of immune inflammatory molecules, especially the decline of IL-6 and NFKB p65 / Rela $(P<0.05)$. Levels of the mRNA relative expression of oxidative stress indicators (Nox2 and Nox4) were only an increasing trend in arterial tissue homogenate of HHcy-associated hypertensive rats, and FA treatment group did not show significant change in the mRNA levels of oxidative stress indicators $(P>0.05)$. Levels of the mRNA relative expression of TGF- $\beta 1$ and TGF- $\beta 3$ also showed an increasing trend in arterial tissue homogenate of HHcy-associated hypertensive rats, and the change of mRNA was also not obvious in FA treatment group $(P>0.05)$. Levels of the mRNA relative expression of adhesion factors 
(MCP-1 and VAP-1) also showed an increasing trend in arterial tissue homogenate of HHcy-associated hypertensive rats, and the change of mRNA was also not obvious in FA treatment group $(P>0.05)$. (Fig. 3)

The protein relative expression levels of immune inflammation indicators (TNF- $a$ and IL-6) were significantly increased in arterial tissue homogenate of $\mathrm{HHcy}$-associated hypertensive rats $(P<0.05)$. But the protein relative expression of IL- 6 showed significant reductions in FA treatment group $(P<0.05)$. The protein relative expression level of oxidative stress indicator Nox2 was also significantly increased in arterial tissue homogenate of $\mathrm{HHcy}$-associated hypertensive rats $(P<0.05)$. And the protein relative expression of Nox2 also showed significant reductions in FA treatment group $(P<0.05)$. (Fig. 4)

\section{Discussion}

Studies have found that HHcy has a close relation with a variety of diseases ${ }^{[25]}$, such as cardiovascular disease, kidney disease, brain parenchymal disease, metabolic disease, and even tumors. The development of disease in HHcy involves endothelial dysfunction, thrombosis, thickening of blood vessel walls, oxidative stress, inflammatory responses, metabolic syndrome, and changes in disease-associated small mRNA ${ }^{[26]}$. Hcy is the intermediate product of methionine metabolism and transsulfuration ${ }^{[14]}$. An abnormal upstream methionine cycle affects the essential methylation process, leading to the high expression of disease-associated small mRNA, while aberrant downstream transsulfuration affects sulfide metabolism, causing abnormalities in gas signal molecules and the redox state. In this study, WKYs and SHRs were administered DL-Hcy intraperitoneally to establish HHcy and hypertension associated with HHcy models. Based on the Hcy-protein antigen theory proposed by Fergusond ${ }^{[27]}$ and the conclusion of Jakubowskie ${ }^{[28,29]}$ that antigen-antibody interactions persist on the intima, this study aims to evaluate the hypothesis that HHcy aggravates vascular damage in hypertension associated with $\mathrm{HHcy}$ mainly through immune/inflammatory mechanisms.

Based on the premise that HHcy induces the abnormal activation of Hcy-protein antigen, which subsequently triggers an immune/inflammatory response, this study investigates, at the molecular level, the role of immunoinflammation and oxidative stress in the pathogenic mechanism of HHcy. We have found that vascular inflammatory factors such as TNF-a and IL- 6 and classical immune/inflammatory pathway indicators such as NF-kB p65/Rela and NF- $\mathrm{KB}_{2}$ were much higher at the mRNA levels in HHcyassociated hypertensive rats compared to the WKYs $(P<0.05)$; The mRNA relative expression of IL- 6 and $\mathrm{NF}-\mathrm{KB}$ in HHcy-associated hypertensive rats was also significantly higher than that in SHRs $(P<0.05)$. Additionally, vascular protein relative expression of TNF-a and IL- 6 in HHcy-associated hypertensive rats was notably increased compared with that in normotensive rats $(P<0.05)$; and IL-6 protein expression in HHcy-associated hypertensive rats was also higher than that in SHRs $(P<0.05)$. We also studied the oxidative stress indicators of the Nox family ${ }^{[30]}$, which are highly expressed in blood vessels. The mRNA relative expression of Nox2 and Nox4 in HHcy-associated hypertensive rats trended towards an increase compared to that in normotensive rats but was not significantly higher than that in SHRs (P凶0.05). Similarly, vascular Nox2 protein relative expression in HHcy-associated hypertensive rats was higher than 
that in normotensive rats but was not significantly different from that in SHRs (P凶0.05). Therefore, HHcy significantly stimulates the intramural immune/inflammatory response, especially the NF-kB pathway, thereby aggravating arterial vascular injury, rather than directly increasing the expression of oxidative stress pathway molecules.

We also analyzed and selectively measured indicators for oxidative stress and inflammation in the blood circulation of HHcy-associated hypertensive rats and found that compared with SHRs, HHcy-associated hypertensive rats manifested higher levels of MDA, a serum oxidative product $(P=0.004)$, and lower levels of SOD, a serum antioxidant molecule $(P=0.000)$, suggesting that $\mathrm{HHcy}$ is associated with increased oxidative stress, which is in line with the pathogenic phenomena that Guo $\mathrm{G}^{[7]}$ and Chan $S^{[8]}$ observed in HHcy. Previous studies also demonstrated that inflammatory indicators such as C-recreative protein ${ }^{[11]}$ and the neutrophil/lymphocyte ratio ${ }^{[10]}$ were significantly higher in patients with HHcy, which suggested that immunoinflammation did participate in the pathogenesis of hypertension associated with HHcy. The above findings are pathological phenomena seen in HHcy or hypertension associated with $\mathrm{HHcy}$, rather than sufficient explanations or evidence regarding whether HHcy directly stimulates oxidative stress or induces inflammation; they also do not provide clues about which plays the dominant role, inflammation or oxidative stress? Thus, more convincing exploration at the molecular level is needed.

The persistent antigen-antibody interaction on the vascular intima inferred from the abnormal Hcy-protein antigen theory in the literature ${ }^{[31-33]}$ and the stronger expression of immune/inflammatory molecules than oxidative stress pathway molecules observed in our study both support the HHcy-induced immune/inflammatory response playing a dominant role in vascular damage of hypertension associated with $\mathrm{HHcy}$. Therefore, we performed immunohistochemical tests for $\mathrm{CD}_{68}$ (an immune cell positive marker) and VAP-1 in the blood vessels in each group of rats and found that the arterial wall of HHcyassociated hypertensive rats had slightly more $\mathrm{CD}_{68}$-positive cells $(\mathrm{P} \otimes 0.05)$ and much higher VAP-1 expression $(P<0.05)$ than did other groups, including the SHR group $(P=0.001)$. These results suggest that the expression of immune cells and immune cell adhesion factors in the arterial wall increases during hypertension associated with HHcy, which supports our hypothesis.

Viel $\mathrm{EC}^{[4]}$ proposed that the degree of infiltration of immune cells is associated with the degree of blood pressure elevation. So, we compared the blood pressure between SHR group and HHcy + SHR group of more immune cell infiltration and inflammation. The SBP and DBP of HHcy-associated hypertensive rats were slightly higher than those of SHRs, but the difference was not statistically significant. We also compared the blood pressure in HHcy and WKY group and obtained similar results. We reviewed the relevant literature about the relation between $\mathrm{HHcy}$ and blood pressure. Some authors ${ }^{[34-38]}$ stated that HHcy elevated blood pressure and was a pathogenic or risk factor for hypertension, while others ${ }^{[39]}$ argued that HHcy could not raise blood pressure. Our findings show that HHcy seems to influence blood pressure but does not significantly increase it. We analyzed the possible reasons: the experimental period of our animal models was only 4 months, which is not enough time to mimic the end-stage vascular 
events of hypertension associated with HHcy; because of the sample size, our study does not have the same statistical impact that large clinical trials provide; thus, significantly elevated blood pressure caused by HHcy was not observed. The pathological analysis of blood vessels with HE and Masson stains also demonstrated that there was no noticeable difference between HHcy-associated hypertensive rats and SHRs. The arterial vascular pathology results for each group also support the above analysis.

We also studied the mechanism of FA's protective effects on the cardiovascular and cerebrovascular systems in hypertension associated with HHcy. Our findings demonstrate that FA does reduce serum Hcy; however, it cannot completely counteract HHcy from external sources; that is, the Hcy level in HHcy + SHR + FA group was still higher than that in the SHR group $(P<0.05)$, suggesting that the FA-dependent transfer of methyl groups, which promotes Hcy's conversion into methionine, can only partially reduce the negative effects of HHcy. As it is known that FA has antioxidant effects, we explored changes in oxidative stress levels and found that FA intervention in HHcy-associated hypertensive rats significantly reduced serum MDA levels $(P=0.000)$ and significantly increased serum SOD levels $(P=0.000)$; SOD was elevated so remarkably that it exceeded the baseline levels in the SHR group $(P=0.000)$. We also tested the effects of FA on oxidative stress and inflammatory responses at the molecular level and found that FA reduced the mRNA relative expression of IL-6 and NF-KB p65/Rela in HHcy-associated hypertensive rats $(P<0.05)$, but did not change the mRNA relative expression of Nox2 and Nox4 (Pख0.05). Experiments showed that the protein level of IL-6 and Nox2 was significantly reduced in HHcy + SHR + FA group $(P<0.05)$. The above findings suggest that the strong antioxidant effects of FA may not be entirely dependent on inhibiting molecules in the main oxidative stress pathway; there may be another independent antioxidative stress pathway that plays a role, such as the nuclear factor erythroid2-related factor2 (Nrf-2)/heme oxygenase-1(HO-1) pathway. Our team is currently studying how FA helps antioxidant stress via the Nrf2/HO-1 pathway. Meanwhile, we have also found that FA can regulate immune/inflammatory response, but the mechanism remains unknown and needs to be explored by further experiments.

In short, FA has a unique effect as a compound preparation for the treatment of hypertension associated with HHcy. FA can both fight HHcy to reduce its negative effects, and also protect tissue cells from damage through independent antioxidant capacity. In addition, FA may also regulate immune cells and inhibit the immune/inflammatory response that might play a leading role in the vascular damage of hypertension associated with HHcy.

The limitations of this study are that the experimental period for the HHcy model did not provide enough time to mimic the end-stage vascular damage in hypertension associated with HHcy; therefore, significant difference in the vascular pathology of HHcy-associated hypertensive rats and SHRs were not detected. There may also be sampling bias regarding vascular pathology, PCR, and western blot $(n=3)$, which affects the interpretation of the findings. The independent antioxidant effects of FA require further exploration at the molecular level. The potential effects of FA on immune/inflammatory response is a new research direction that we have discovered and requires verification by further research. 


\section{Conclusion}

Immune/inflammatory response plays a leading role in arterial vascular pathology of hypertension associated with HHcy. The classic inflammatory pathway molecule NF-kB p65 / Rela can still be used as an intervention target for immune regulation. IL- 6 can be a reliable observation target for immune/inflammatory response in hypertension associated with HHcy. FA has unique therapeutic value for HHcy, showing strong antioxidant capacity and potential immunomodulatory effects.

\section{Declarations}

\section{Acknowledgements}

We special thank Haixia Liu for her help with the histopathology and immunohistochemistry.

\section{Author contributors}

LHZ and RX designed the experiment; LHZ, Changcheng Xing, Ning Gao, Ping Cao, Qingcheng Hui performed the experiments; ZLL analyzed the data and prepared the figures; LHZ and LY wrote the manuscript; and RX had primary responsibility for the final content. All authors read and approved the final manuscript.

\section{Ethics approval and consent to participate}

Animal care and experimental protocol for this study were approved by the Committee on the Use of Live Animals in Teaching and Research of Qianfoshan Hospital.

\section{Consent for publication}

All participants in this experiment agree and hope to publish the manuscript.

\section{Availability of data and materials}

I solemnly declare that the research data and experimental materials are true and reliable.

\section{Competing interests}

No conflicts of interest, financial or otherwise, are declared by the authors.

\section{Funding}

This study was supported by grants from Shandong Provincial Key Research and Development Programme Foundation, China (Grant No. 2018GSF118009), Technology Programme Foundation of Jinan, China (Grant No. 201821007) $₫$ Shandong Provincial Medical Science and Technology Development Programme Foundation, China (Grant No. 2017WS462) and Jinan City Medical and Health Development Plan(Grant No. 2018-2-31) 


\section{References}

1. Hu DY, Xu XP. Prevention of stroke relies on valid control "H" type hypertension[J]. Zhonghua nei ke za zhi. [Chinese journal of internal medicine]. 2009;47(12):976-7.

2. Jian-ping $L$, Yong $H$, Ping L, et al. Efficacy and safety of Enalapril-Folate acid tablets in lowering blood pressure and plasma homocysteine[J]. JOURNAL OF PEKING UNIVERSITY(HEALTH SCIENCES, 2007,39(6):614-618.

3. Warso MA. Presence of lipid hydroperoxide in human plasma.[J]. Journal of Clinical Investigation. 1985;75(2):667-71.

4. Viel EC, Lemarié CA, Benkirane $K$, et al. Immune regulation and vascular inflammation in genetic hypertension.[J]. Am J Physiol. 2010;298(2):938-44.

5. Graham IM, Daly LE, Refsum HM, et al. Plasma Homocysteine as a Risk Factor for Vascular Disease: The European Concerted Action Project[J]. Jama. 1997;277(22):1775-81.

6. Woo KS, Chook P, Lolin YI, et al. Hyperhomocyst(e)inemia is a risk factor for arterial endothelial dysfunction in humans.[J]. Circulation. 1997;96(8):2542-4.

7. Guo G, Sun W, Liu G, et al. Comparison of oxidative stress biomarkers in hypertensive patients with or without hyperhomocysteinemia[J]. Clinical \& Experimental Hypertension, 2018:1-5.

8. Chan $\mathrm{SH}$, Hung $\mathrm{CH}$, Shih JY, et al. Exercise intervention attenuates hyperhomocysteinemia-induced aortic endothelial oxidative injury by regulating SIRT1 through mitigating NADPH oxidase/LOX-1 signaling[J]. 2018.

9. Dayal S, Baumbach GL, Arning E, et al. Deficiency of superoxide dismutase promotes cerebral vascular hypertrophy and vascular dysfunction in hyperhomocysteinemia[J]. Plos One. 2017;12(4):e175732.

10. Gang L, Zhang Y. Increased neutrophil to lymphocyte ratio in persons suffering from hypertension with hyperhomocysteinemia[J]. Hypertension Research Official Journal of the Japanese Society of Hypertension. 2016;39(8):606.

11. Cao L G Y Z Z. Study of the Inflammatory Mechanisms in Hyperhomocysteinemia on Large-Artery Atherosclerosis Based on Hypersensitive C-Reactive Protein-A Study from Southern China[J]. Journal of Stroke Cerebrovascular Diseases, 2019,8(32):1101-4.

12. Celik SFCE. Subclinical atherosclerosis and impaired cardiac autonomic control in pediatric patients with Vitamin B12 deficiency[J]. NIGER J CLIN PRACT, 2018(21):1012-1016.

13. Chen Z, Wang F, Zheng $Y$, et al. H-type hypertension is an important risk factor of carotid atherosclerotic plaques[J]. Clinical Experimental Hypertension. 2016;38(5):424-8.

14. Henrieta K, Eva V, Silvia M, et al. The Molecular and Cellular Effect of Homocysteine Metabolism Imbalance on Human Health[J]. Int J Mol Sci. 2016;17(10):1733.

15. Glowacki R. Cross-talk between Cys34 and Lysine Residues in Human Serum Albumin Revealed by NHomocysteinylation[J]. J Biol Chem. 2004;279(12):10864-71. 
16. Per A-Kajan J, Utyro O, Rusek M, et al. N-Homocysteinylation impairs collagen cross-linking in cystathionine $\beta$-synthase-deficient mice: a novel mechanism of connective tissue abnormalities[J]. Faseb Journal, 2016:201600539.

17. Akchiche N, Bossenmeyer-Pourie C, Kerek R, et al. Homocysteinylation of neuronal proteins contributes to folate deficiency-associated alterations of differentiation, vesicular transport, and plasticity in hippocampal neuronal cells[J]. Faseb Journal. 2012;26(10):3980-92.

18. Wang YJTS. Hyperhomocysteinemia-mediated DNA Hypomethylation and its Potential Epigenetic Role in Rats[J]. Acta Biochimica Et Biophysica Sinica. 2007;039(009):657-67.

19. Fox, Stover JT. P. J. Folate-mediated one-carbon metabolism[J]. Vitamins Hormones-advances in Research Applications. 2008;79(08):1-44.

20. Verhaar MC, Wever RMF, Kastelein JJP, et al. 5-Methyltetrahydrofolate, the Active Form of Folic Acid, Restores Endothelial Function in Familial Hypercholesterolemia[J]. Circulation. 1998;97(3):237-41.

21. Ofer S. And, et al. Identification of two putative novel folate receptor genes in humans and mouse[J]. 2000.

22. Xia W, Hilgenbrink AR, Matteson EL, et al. A functional folate receptor is induced during macrophage activation and can be used to target drugs to activated macrophages[J]. Blood. 2008;113(2):438-46.

23. Wang B, Lin L, Zhao C. Related factors of serum uric acid in patients with primary hypertension and hyperhomocysteinemia[J]. Clinical Experimental Hypertension. 2016;38(3):312-6.

24. Durand P, Lussier-Cacan S, Blache D. Acute methionine load-induced hyperhomocysteinemia enhances platelet aggregation, thromboxane biosynthesis, and macrophage-derived tissue factor activity in rats.[J]. Faseb Journal. 1997;11(13):1157-68.

25. Kim J, Kim H, Roh H, et al. Causes of hyperhomocysteinemia and its pathological significance[J]. Archives of Pharmacal Research, 2018.

26. Fu Y, Wang X, Kong W. Hyperhomocysteinaemia and vascular injury: advances in mechanisms and drug targets[J]. British Journal of Pharmacology, 2018,175(8).

27. Ferguson E, Parthasarathy S, Joseph J, et al. Generation and initial characterization of a novel polyclonal antibody directed against homocysteine thiolactone-modified low density lipoprotein.[J]. $1998,39(4): 925-933$.

28. Jakubowski H. Anti-N-homocysteinylated protein autoantibodies and cardiovascular disease[J]. Clinical Chemistry Laboratory Medicine. 2005;43(10):1011-4.

29. Hieronim J. Pathophysiological Consequences of Homocysteine Excess[J]. Journal of Nutrition, 2006(6):6.

30. Bedard K, Krause KH. The NOX family of ROS-generating NADPH oxidases: physiology and pathophysiology.[J]. Physiological Reviews, 2007.

31. Ferguson E, Parthasarathy S, Joseph J, et al. Generation and initial characterization of a novel polyclonal antibody directed against homocysteine thiolactone-modified low density lipoprotein.[J]. $1998,39(4): 925-933$. 
32. Undas A, Jankowski M, Twardowska M, et al. Antibodies to N-homocysteinylated albumin as a marker for earlyonset coronary artery disease in men[J]. Thrombosis Haemostasis. 2005;93(02):346-50.

33. Rosanna C, Irene S, Amelia C, et al. Homocysteinylated Albumin Promotes Increased MonocyteEndothelial Cell Adhesion and Up-Regulation of MCP1, Hsp60 and ADAM17[J]. Plos One. 2012;7(2):e31388.

34. Nygård O, Vollset SE, Refsum H, et al. Total Plasma Homocysteine and Cardiovascular Risk Profile: The Hordaland Homocysteine Study[J]. Jama. 1995;274(19):1526.

35. Osganian SK, Stampfer MJ, Spiegelman D, et al. Distribution of and Factors Associated With Serum Homocysteine Levels in Children[J]. Jama. 1999;281(13):1189.

36. N S, T L. C. S, et al. Total plasma homocysteine is associated with hypertension in Type I diabetic patients[J]. Diabetologia. 2002;45(9):1315-24.

37. Kahleová R, Palyzová D, Zvára K, et al. Essential hypertension in adolescents: association with insulin resistance and with metabolism of homocysteine and vitamins[J]. Am J Hypertens. 2002;15(10 Pt 1):857-64.

38. Lim UCP. Homocysteine and blood pressure in the third National Health and Nutrition Examination Survey, 1988-1994[J]. Am J Epidemiol, 2002(156):1105-1113.

39. Guldener CV, Nanayakkara PWB, Stehouwer CDA. Homocysteine and blood pressure[J]. Curr Hypertens Rep. 2003;5(1):26-31.

\section{Tables}


Table 1

Primer sequences for quantitative real-time polymerase chain reaction (qRT-PCR) analysis from Takara.

\begin{tabular}{|lll|}
\hline Name & Sequence $-\mathbf{F}$ & Sequence $-\mathbf{R}$ \\
\hline VAP-1 & CACACACTGGGCACTGTACACAC & CCGGTTGCCAAGGTACATTCATA \\
\hline TNF-a & GGCGTGTTCATCCGTTCTC & CTTCAGCGTCTCGTGTGTTTCT \\
\hline IL-6 & ATTGTATGAACAGCGATGATGCAC & CCAGGTAGAAACGGAACTCCAGA \\
\hline NOX2 & CCTGGAGACCCAGATGCAAGA & CGTGGTGCACAGCAAAGTGA \\
\hline NOX4 & ACTGGTGAAGATTTGCCTGGAAG & CACAGTATAGGCACAAAGGTCCAGA \\
\hline TGF- $\beta_{1}$ & CATTGCTGTCCCGTGCAGA & AGGTAACGCCAGGAATTGTTGCTA \\
\hline TGF- $\beta_{3}$ & CCCTGGACACCAATTACTGCTTC & CCTTAGGTTCGTGGACCCATTTC \\
\hline NF-KB p65/Rela & ATCCCTGCTTCCCCTTTCTC & CTGTCTTATGGCTGAGGTCTGGT \\
\hline MCP-1 & CTATGCAGGTCTCTGTCACGCTTC & CAGCCGACTCATTGGGATCA \\
\hline NF-KB 2 & CTGATGGCACAGGACGAGAA & TGGGCTATCTGCTCAATGACAC \\
\hline
\end{tabular}

Table 2

The levels of homocysteine (Hcy), malondialdehyde (MDA), superoxide dismutase (SOD) and systolic blood pressure (SBP), diastolic blood pressure (DBP) in Wistar-Kyoto (WKY), hyperhomocysteinemia (HHcy), spontaneously hypertensive rat (SHR), HHcy + SHR and HHcy + SHR + folic acid (FA) group. Values represent means \pm SD ( ${ }^{\star} P<0.05$ vs. WKY group, ${ }^{\$} P<0.05$ vs. SHR group, ${ }^{\&} P<0.05$ vs. HHcy $+S H R$ group, $\mathrm{n}=8$ )

\begin{tabular}{|c|c|c|c|c|c|}
\hline group & $\begin{array}{l}\text { WKY } \\
(n=8)\end{array}$ & $\begin{array}{l}\text { HHcy } \\
(n=8)\end{array}$ & $\begin{array}{l}\text { SHR } \\
(n=8)\end{array}$ & $\begin{array}{l}\text { HHcy + SHR(n } \\
=8)\end{array}$ & $\begin{array}{l}\text { HHcy + SHR + } \\
\text { FA(n = 8) }\end{array}$ \\
\hline $\mathrm{Hcy}(\mu \mathrm{mol} / \mathrm{L})$ & $6.30 \pm 1.47$ & $\begin{array}{l}25.48 \pm \\
2.01^{*}\end{array}$ & $8.26 \pm 1.77^{\star}$ & $27.61 \pm 1.53^{\star \$}$ & $10.93 \pm 2.34^{\star \$ \&}$ \\
\hline $\operatorname{MDA}(\mathrm{nmol} / \mathrm{mL})$ & $5.37 \pm 0.85$ & $6.50 \pm 1.07^{\star}$ & $7.63 \pm 0.70 *$ & $9.11 \pm 1.12^{\star \$}$ & $6.72 \pm 1.05^{\star \&}$ \\
\hline $\mathrm{SOD}(\mathrm{U} / \mathrm{mL})$ & $\begin{array}{l}516.85 \pm \\
28.69\end{array}$ & $\begin{array}{l}479.64 \pm \\
18.38^{*}\end{array}$ & $\begin{array}{l}432.50 \pm \\
19.73^{*}\end{array}$ & $\begin{array}{l}362.73 \pm \\
30.23 * \$\end{array}$ & $479.04 \pm 20.62 * \$ \&$ \\
\hline $\mathrm{SBP}(\mathrm{mmHg})$ & $\begin{array}{l}119.25 \pm \\
11.85\end{array}$ & $\begin{array}{l}121.88 \pm \\
9.89\end{array}$ & $\begin{array}{l}205.13 \pm \\
9.54^{*}\end{array}$ & $209.75 \pm 9.81^{*}$ & $205.25 \pm 9.45^{*}$ \\
\hline $\mathrm{DBP}(\mathrm{mmHg})$ & $\begin{array}{l}81.13 \pm \\
4.32\end{array}$ & $86.63 \pm 6.80$ & $\begin{array}{l}134.88 \pm \\
6.73^{*}\end{array}$ & $140.63 \pm 7.39^{*}$ & $146.63 \pm 11.50^{\star \$}$ \\
\hline
\end{tabular}


Figures

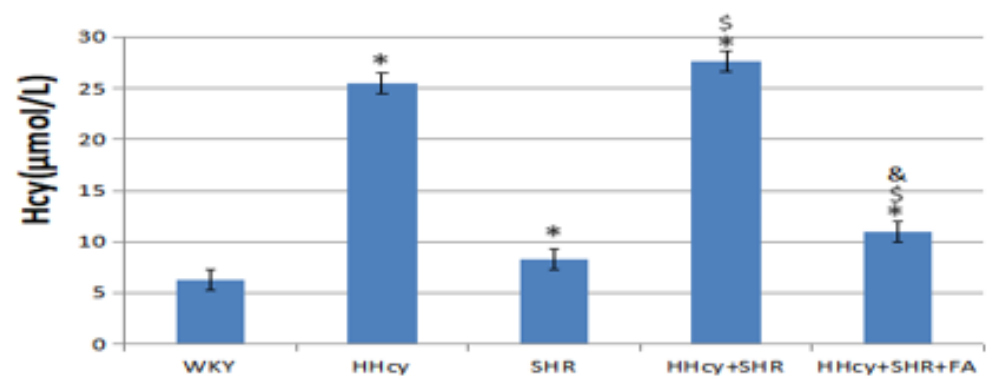

Fig 1-A Changes of Hcy in rats of all groups.

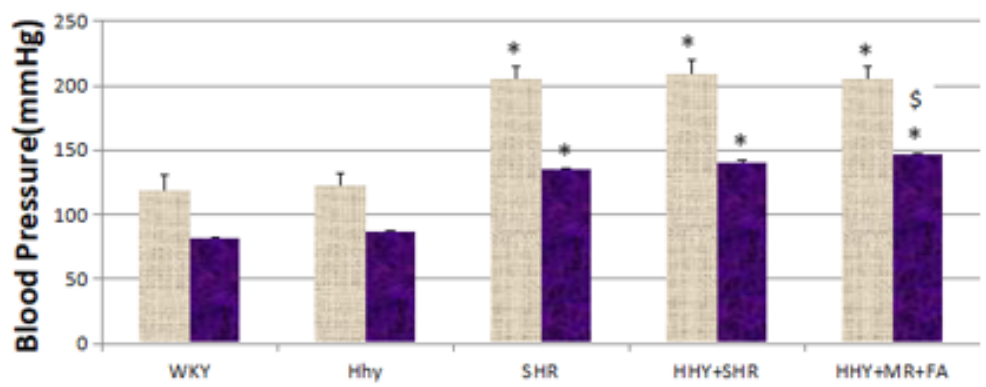

Fig 1-B Changes of blood pressure in rats of all groups.

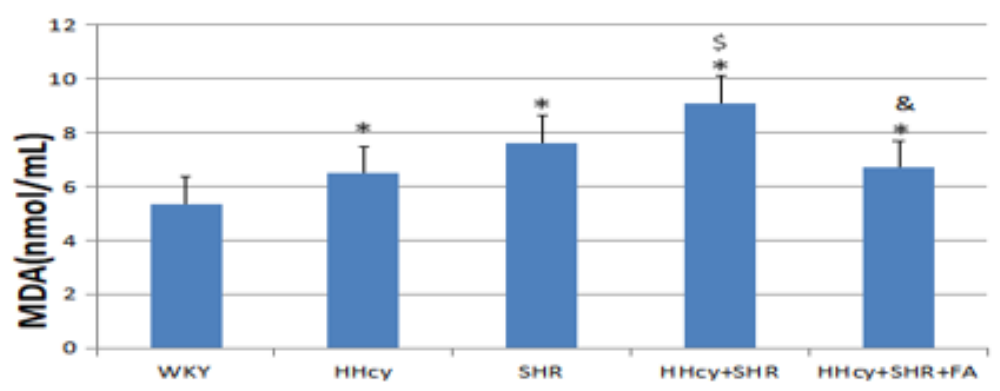

Fig 1-C Changes of MDA in rats of all groups.

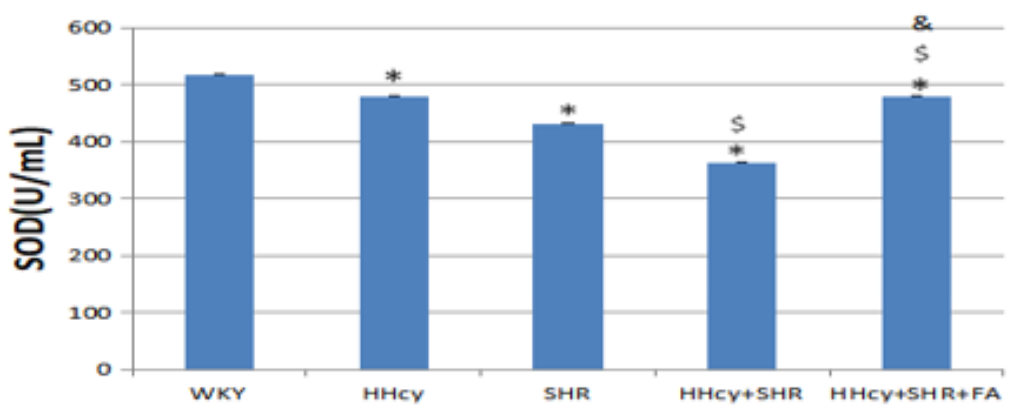

Fig 1-D Changes of blood SOD in rats of all groups.

Figure 1 
The levels of homocysteine (Hcy), superoxide dismutase (SOD) and malondialdehyde (MDA), systolic blood pressure (SBP), diastolic blood pressure (DBP) in Wistar-Kyoto (WKY), hyperhomocysteinemia (HHcy), spontaneously hypertensive rat (SHR), HHcy + SHR and HHcy + SHR + folic acid (FA) group. (A) the levels of Hcy in WKY, HHcy, SHR, HHcy + SHR and HHcy + SHR + FA group. (B) the levels of SBP (tan) and DBP(blue) in WKY, HHcy, SHR, HHcy + SHR and HHcy + SHR + FA group. (C) the levels of MDA in WKY, HHcy, SHR, HHcy + SHR and HHcy + SHR + FA group. (D) the levels of SOD in WKY, HHcy, SHR, HHcy + SHR and HHcy + SHR + FA group. Values represent means $\pm S D\left({ }^{*}<<0.05\right.$ vs. WKY group, $\$ \mathrm{P}<$ 0.05 vs. SHR group, $\& P<0.05$ vs. HHcy + SHR group, $n=8$ )

A
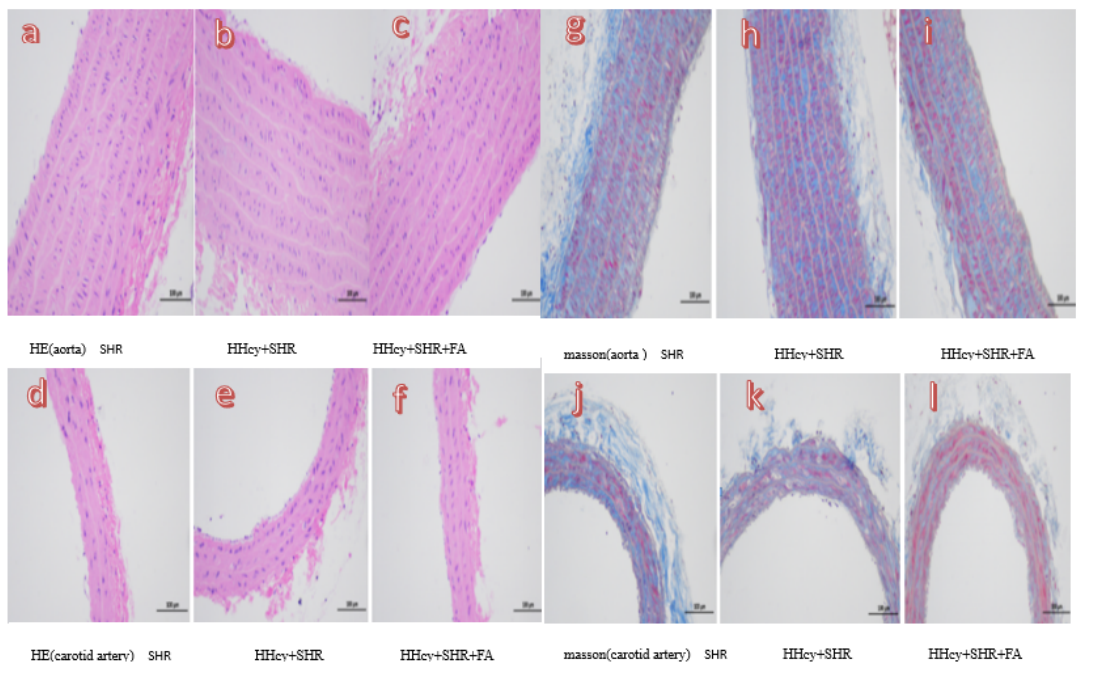

$\mathrm{C}$
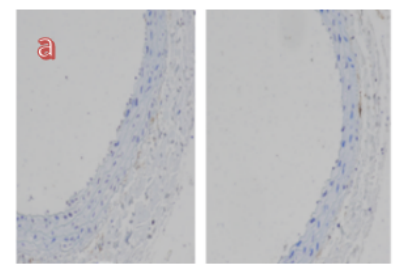

HHcy

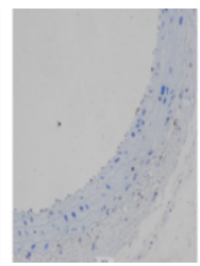

SHR

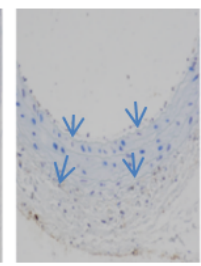

HHcy+SHR
$\mathrm{B}$

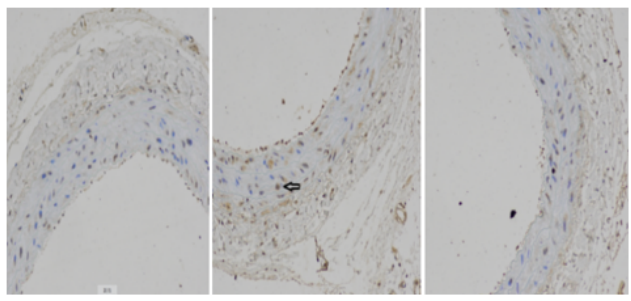

HHcy+SHR

HHcy + SHR $+\mathrm{FA}$

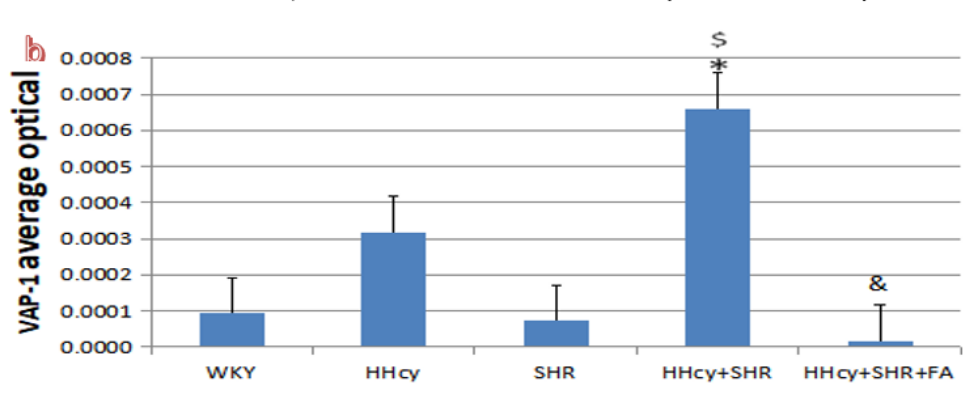

\section{Figure 2}

A. HE and masson staining of aorta and carotid artery in spontaneously hypertensive rat (SHR), hyperhomocysteinemia (HHcy) + SHR and HHcy + SHR + folic acid (FA) group. (a)邓(b) and (c) represent 
HE staining of rat aorta; (d)凶(e) and (f) represent HE staining of rat carotid artery; (g) $)(\mathrm{h})$ and (i) represent

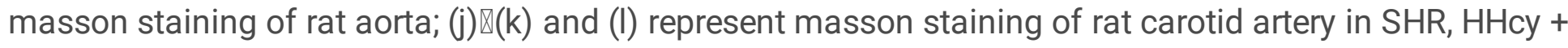
SHR and HHcy + SHR + FA group respectively. HE staining of aorta (b) and carotid artery (e) showed the thickening of the vascular smooth muscle layer compared with (a) and (d) respectively. Masson staining of aorta $(\mathrm{h})$ and carotid artery $(k)$ showed the deposition of collagen fibers compared with $(\mathrm{g})$ and $(\mathrm{j})$ respectively. Pathological damage was slightly mitigated in HHcy + SHR + FA group (c, f, i, l) compared

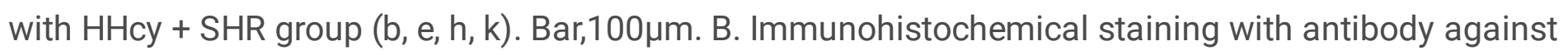
CD68 in spontaneously hypertensive rat (SHR), hyperhomocysteinemia (HHcy) + SHR and HHcy + SHR + folic acid (FA) group. Immunostaining of rat carotid artery showed slightly more CD68 cell (arrowhead) in

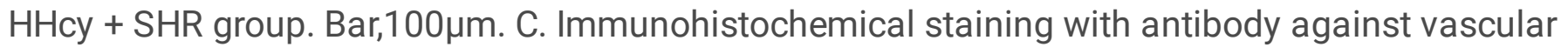
adhesion protein-1 (VAP-1) in Wistar-Kyoto (WKY), hyperhomocysteinemia (HHcy), spontaneously hypertensive rat (SHR), HHcy + SHR and HHcy + SHR + folic acid (FA) group. Immunostaining of rat carotid artery (a) showed that the expression of VAP-1 (arrowheads) was significantly increased in HHcy + SHR group. And the average optical (AO) value of VAP-1 (b) was also significantly increased in HHcy + SHR group $(\mathrm{P}<0.05)$. But the AO value of VAP-1(b) was significantly reduced in HHcy + SHR + FA group $(P=0.001)$. Values represent means $\pm S D\left({ }^{*} P<0.05\right.$ vs. WKY group, $\$ P<0.05$ vs. SHR group, $\& P<0.05$ vs. HHcy + SHR group, $n=3$ ) 

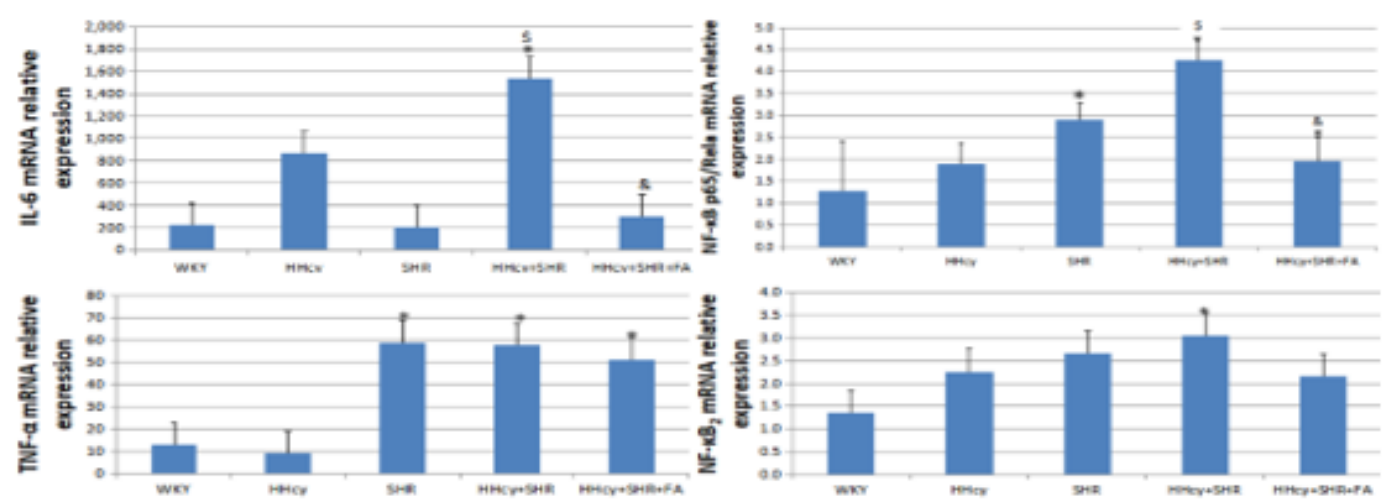

Fig 3-A mRNA relative expression of immune inflammatory factors in rats of all groups.
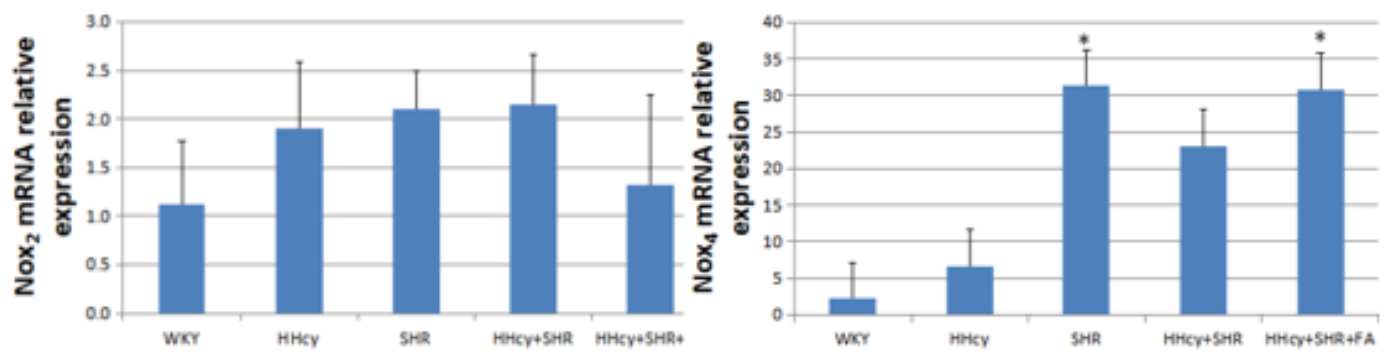

Fig 3-B mRNA relative expression of $\mathrm{NOX}_{2}$ and $\mathrm{NOX}_{4}$ in rats of all groups.
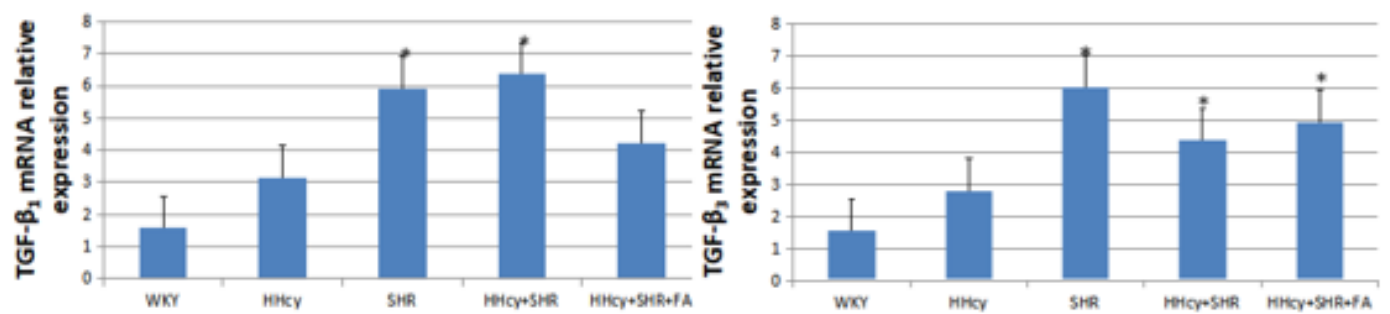

Fig 3-C mRNA relative expression of TGF- $\beta 1$ and TGF- $\beta 3$ in rats of all groups.
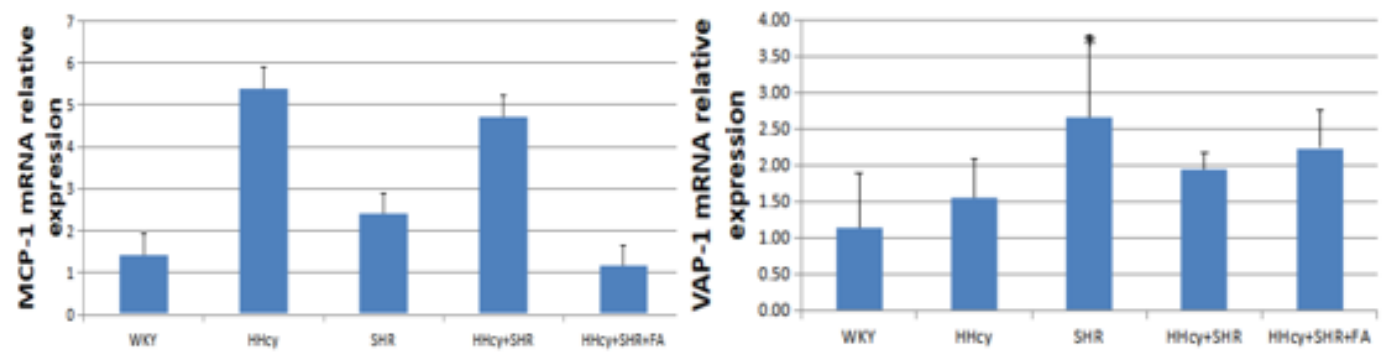

Fig 3-D mRNA relative expression of MCP-1 and VAP-1 in rats of all groups.

\section{Figure 3}

The relative mRNA expressions in rat aortic tissues $(n=3)$. (A) relative mRNA levels of TNF- $a, I L-6, N F-K B$ p65 / Rela, and NF-KB2 by quantitative real-time polymerase chain reaction (qRT-PCR) analysis in rat aortic tissues among Wistar-Kyoto (WKY), hyperhomocysteinemia (HHcy), spontaneously hypertensive rat (SHR), HHcy + SHR and HHcy + SHR + folic acid (FA) group. (B) relative mRNA levels of NOX2 and NOX4 by qRT-PCR analysis. (C) relative mRNA levels of transforming growth factors(TGF)- $\beta 1$ and TGF- $\beta 3$ 
by qRT-PCR analysis. (D) relative mRNA levels of monocyte chemotactic protein-1 (MCP-1) and vascular adhesion protein-1 (VAP-1) by qRT-PCR analysis. Values represent means $\pm S D\left({ }^{*} P<0.05\right.$ vs. WKY group, $\$ P<0.05$ vs. SHR group, \&P< 0.05 vs. HHcy + SHR group, $n=3$ )
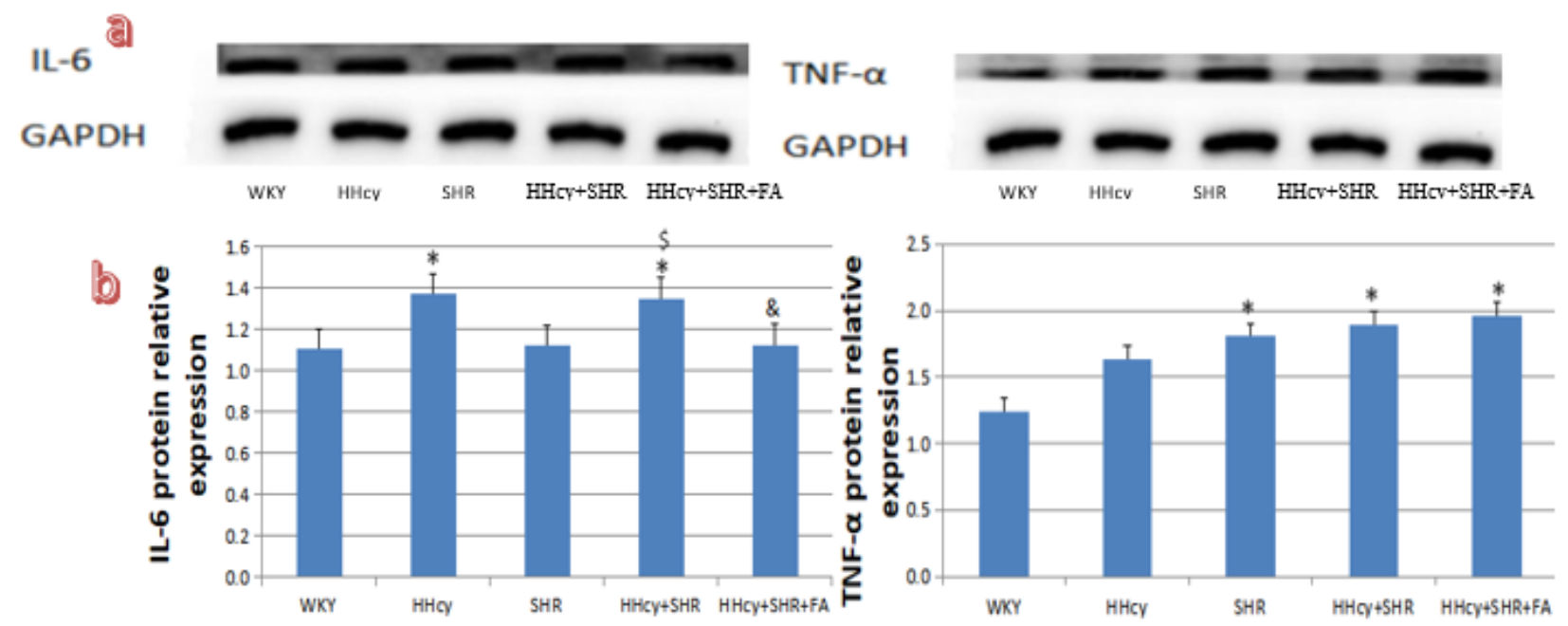

Fig 4-A Protein expression levels of immune inflammatory factors in rats of all groups.
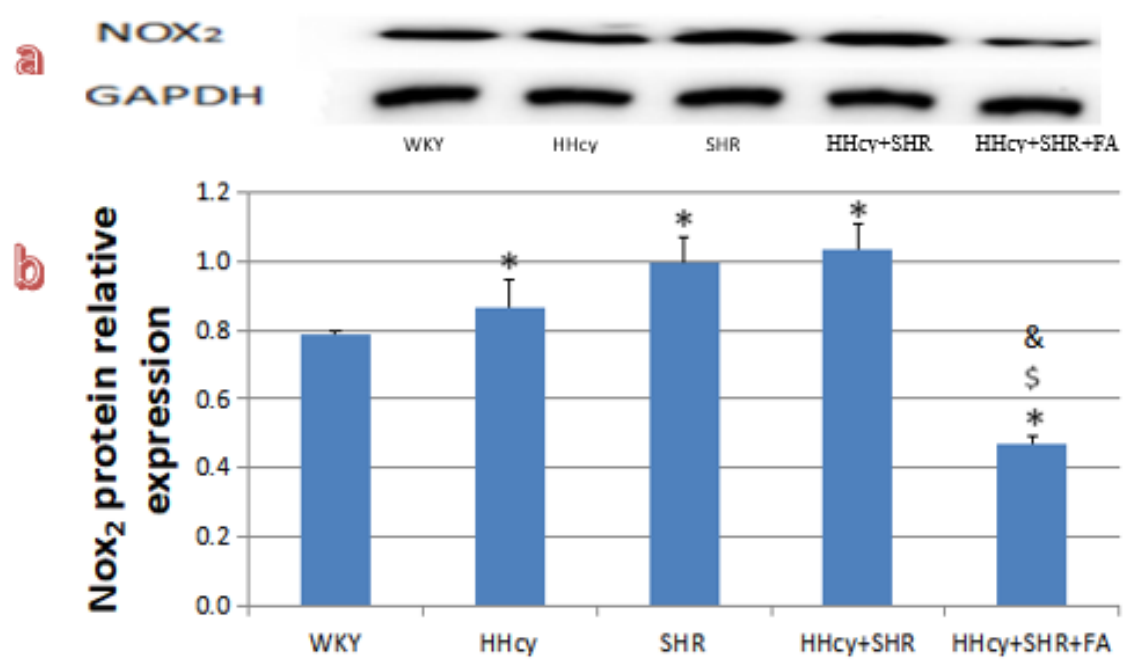

\section{Figure 4}

The relative protein levels in rat aortic tissues $(n=3)$. (A) western blot analyses of IL- 6 and TNF-a protein levels in rat aortic tissues, includes representative blot images and densitometric summary of the blot analysis after normalized to GAPDH among Wistar-Kyoto (WKY), hyperhomocysteinemia (HHcy), spontaneously hypertensive rat (SHR), HHcy + SHR and HHcy + SHR + folic acid (FA) group. (B) western blot analyses of NOX2 protein levels in rat aortic tissues, includes representative blot images and densitometric summary of the blot analysis after normalized to GAPDH. Values represent means \pm SD $\left({ }^{*} \mathrm{P}<0.05\right.$ vs. WKY group, $\$ \mathrm{P}<0.05$ vs. SHR group, $\& \mathrm{P}<0.05$ vs. HHcy + SHR group, $\left.n=3\right)$ 